\title{
LAS POLÍTICAS DEL NOMBRE PROPIO \\ Dilemas de representación en las Ciencias Sociales
}

\section{Carmen Ascanio-Sánchez ${ }_{(1)}$}

http://orcid.org/0000-0002-0299-1958

\section{Barbara Rostecka}

https://orcid.org/0000-0003-2779-6128

(1) Departamento de Sociología y Antropología de la Universidad de La Laguna (ULL), Islas Canarias, Espanha. E-mail: cascanio@ull.edu.es (2) Departamento de Sociología y Antropología de la Universidad de La Laguna (ULL), Islas Canarias, Espanha. E-mail: brostecka@gmail.com DOI: $10.1590 / 3410112 / 2019$

At the beginning of a new century it is necessary to reengage the promise of qualitative research as a form of radical democratic practice. The narrative turn in the social sciences has been taken. We have told our tales from the field. Today we understand that we write culture, and that writing is not an innocent practice. We know the world only through our representations of it.

Denzin $(2001,23)$

\section{Introducción, metodología y dilemas éticos}

Este trabajo pretende contribuir al debate sobre la representación de los sujetos de estudio en las ciencias sociales y, concretamente, el centrado en las

Artigo recebido em: 15/03/2018

Aprovado em: 15/04/2019 políticas del nombre propio y, por tanto, en el anonimato/identificación o nombramiento. A partir de dos investigaciones empíricas, donde estos asuntos requirieron de especial atención, se debaten las raíces teóricas de dicha práctica y sus implicaciones en la reformulación del método científico, de los sujetos de estudio y de las políticas de representación.

Precisamente a través del trabajo de campo realizado surgieron numerosos interrogantes que pusieron el foco de atención en la producción del conocimiento. Los sujetos de nuestras investigaciones no sólo producían datos sobre aspectos de lo social, sino que iban dejando rastros de sus anhelos, miedos, tristezas..., casi siempre como susurros en comentarios y observaciones, pero, también, con claras alusiones que nos fue llevando a revisar las raíces simbólicas de dichas prácticas, así como sus implicaciones sociales y metodológicas.

El análisis lo articularemos a través de cuestiones clave de tipo metodológico y ético, desarrollándo- 
las a través de los siguientes puntos: a) el anonimato como protocolo; b) el nombrarlidentificar como recurso metodológico de validación; c) el nombre propio como base de procesos de subjetivación y construcción de la identidad y d) la identificación en los procesos de la democratización del espacio de la investigación. Consideramos que estas cuatro cuestiones son representativas de debates actuales en las ciencias sociales, que se centran en una creciente inquietud por renovar marcos epistémicos y ontológicos.

A modo de introducción, se puede afirmar que ha sido el paradigma durkheimiano de los hechos sociales, entendidos estos últimos como transgresión del espacio unitario, privado e íntimo de la persona, el que deroga la idea de identificar a los que nutren de datos nuestras investigaciones. Como contrapunto, la perspectiva subjetivista de origen weberiano plantea que cualquier análisis no es sino una reconstrucción de la conciencia particular (Polanyi, 1966; Popper, 1977) y que las múltiples vertientes de la vida social nunca se han separado de la individualidad: al contrario, se articulan y conforman en base de los complejos procesos de subjetivación y construcción de las identidades.

Esta es una más de las rupturas acaecidas entre el objetivismo y el subjetivismo que ha marcado el desarrollo de la noción del sujeto y de sus epistemes. El desarrollo del espacio público ha ido paralelo al proceso de relativización de la noción del sujeto como héroe histórico (Sztompka, 2002) y, por ende, con el crecimiento de nuevas identidades sociales y de múltiples agencias. Esto se aprecia, de modo especial, en los enfoques decoloniales que han articulado nuevas representaciones sociales a través de reelaborar nociones como "pueblo", "otredad", "colonialismo" etc. (Alarcón, 2001). Este proceso ha sido de especial relevancia en Latinoamérica cuya redefinición epistemológica ha estado fuertemente marcada por el distanciamiento de la investigación con los posmodernos conflictos europeos, lo que les ha permitido identificar nuevos roles entre investigadores e investigados, adentrarse en sus consecuencias para el compromiso social y desarrollar otros ámbitos de atención como, por ejemplo, los procesos de bienestar y dialogo social (Dussel, 1988, Freire, 1988). También ha sido de especial interés para ciertas disciplinas, como la antropología y ciertos enfoques sociológicos, cuyo tipo de conocimiento se ha construido a partir del contacto entre investigador/investigado, a pesar del inicial modelo colonial que presuponía una relación vertical entre ambos.

Hay que señalar que los primeros trabajos sociológicos que usaban los nombres propios como reconocimiento (Thomas y Znaniecki, entre 19181920, Fals Borda en los años cincuenta) han catalogado sus prácticas dentro de investigaciones de sociología histórica o de intervención social. Otros autores han centrado el foco de atención, de modo más amplio, en la autoría de la generación de conocimientos. Foucault (1984) criticaba el papel del autor en la explicación de su obra y declaraba el nuevo orden de micropoderes en el campo de la creación literaria; Derrida (2009) ofrecía herramientas de la deconstrucción de la lectura que consistían en hacer repaso de las correspondencias entre la vida y la obra del autor. Es decir, el proceso de nombrar plantea desafíos que se adentran en los anclajes de la identidad (Revilla, 2003), de la memoria colectiva y de los proyectos de ser social al desvelarse que el nombre singular es:

\section{(...) el conjunto de las posiciones ocupadas si- multáneamente en un momento dado del tiempo por una individualidad lógica socialmente insti- tuida, actuando como soporte de un conjunto de atributos $y$ de atribuciones propias para permitir- le intervenir como agente eficiente en diferentes campos (Bourdieu, 2011, p.128).}

Como se ha señalado, los nuevos paradigmas y dilemas emergentes han estado siempre atravesados por debates sobre la subjetividad, la interdisciplinaridad y las relaciones de poder entre investigadores/ investigados (Marre, 2010). Por este motivo, es relevante visibilizar las prácticas de los investigadores, sobre todo aquellas practicadas bajo el paraguas de lo ético. No se trata de volver a antiguas dicotomías, sino más bien de definir nuevas bases en el quehacer de los científicos sociales, adaptadas a las situaciones contemporáneas y afines a las nuevas sensibilidades (Beck, 2004; Elías, 1994; Dussel, 1988; Flick, 2004; Freire, 1988; Touraine, 2002). 


\section{Instantáneas desde los campos de investigación}

Los dilemas sobre la representación de los sujetos de estudio que han marcado los trabajos de las autoras giran en torno a "la naturaleza de las relaciones entre la subjetividad y la objetividad, entre el actor y el sistema" (Dubet, 2007, p.108) y el rol del investigador en la modulación de cada uno de estos procesos. De acuerdo con Carens (2004), replanteamos aquí el enfoque de tipo contextual que esboza un análisis continuamente retroalimentado desde la teoría a la práctica y viceversa; en la medida de lo que permite este espacio reflejamos el dialogo entre lo teórico y las experiencias empíricas que tuvieron lugar en las Islas Canarias (Ascanio-Sánchez, 2007; Rostecka 2013).

\section{Dilemas y falacias del nombrar (Carmen Ascanio-Sánchez)}

Mis primeros trabajos de campo profesional, finalizada mi formación en antropología social, los realicé en contextos alejados del lugar donde nací y había sido mi residencia habitual. Regresar a la isla de Gran Canaria (Islas Canarias, España), dónde había nacido, supuso un contraste inesperado que me animó a realizar varias investigaciones, una de ellas en mi municipio de origen. Allí pocas personas me conocían físicamente, aunque pronto me di cuenta de que me ubicaban con rapidez si me identificaba a través de mis padres; por tanto, este hecho - el nombrarme o el no nombrarme - se convirtió en una estrategia útil de acceso a barrios, hogares y gentes. Utilizar la identidad de parentesco como forma de cercanía, empatía y construcción de confianza social hizo que me fuera planteando esos usos de la identidad y la confidencialidad.

Poco después, en un barrio del mismo municipio, surgiría la indagación más sugerente a la que me he enfrentado. Comenzó en un lugar... ¿lo nombro?, porque esta fue otra duda, hoy resuelta. Por tanto, comenzó en el barrio de La Atalaya cuando unos conocidos me invitaron a una fiesta popular que, al parecer, rememoraba la tradición alfarera del lugar. Para los oriundos de la zona este era un lugar diferente al resto del municipio, que se explicitaba de modo claro en diversos discursos negativos sobre sus habitantes (carácter, hábitos, comportamientos, etc.) y en la focalidad de un oficio histórico: la fabricación de loza o alfarería. Tras asistir al evento me impactó el pregón de las fiestas que contenía un encendido discurso identitario sobre el lugar y sus gentes. Decidí indagar algunas cuestiones y poco después comenzaría un trabajo de campo etnográfico en el lugar. En poco tiempo, todo lo relacionado con este proceso identitario me remitía a la figura de un alfarero, "el primero" y/o "el "ultimo” según las narrativas, fallecido ya. Según iba consultando fuentes y entrevistando a personas, fui comprobando que este personaje parecía el único nombrado/identificado que no tenía asociados estereotipos negativos y que, además, concentraba de modo intenso memorias del lugar: tanto las de un pasado reciente como las de un pasado más lejano, donde el barrio fue foco de atención entre foráneos y turistas extranjeros.

Cuanto más tiempo pasaba más quiebras ${ }^{1}$ surgían: ¡cómo, en una sociedad dónde el oficio de alfarero ha sido femenino, un hombre ha llegado a ser la principal imagen del oficio?, ¿cómo y por qué se ha permitido esta transgresión? Las posibles respuestas fueron surgiendo desde escritos literarios que lo nombraban, desde fotografías que me entregaban y, especialmente, desde las conversaciones con diversos informantes, entre ellos los discípulos del maestro. Con éstos establecí una intensa relación y por lo que pude apreciar atravesaban una época de especial conflictividad, por motivos que iban de lo patrimonial-económico a lo personal y emocional. Precisamente, fueron estos discípulos del maestro los que fueron más contundentes en sus respuestas, en ocasiones desde la ambigüedad y los eufemismos, pero también desde algunas confesiones, producto de los meses de estancia y de esos ratos de encuentro que, en ocasiones, producen las narrativas más densas de lo que es el alargamiento de un trabajo de campo no estructurado.

En fin, todos estos discursos dirigían su atención a la diferencia sexual del alfarero: ciertamente nunca se nombró un hecho o práctica, pero fui sobreentendiendo, desde múltiples narrativas y silencios, la clara alusión a la homosexualidad del personaje. Y si nadie lo nombraba, etiquetándolo, y yo nunca me 
atreví a plantearlo directamente ¿podía luego exponerlo? Una nueva duda sobre el nombrar: en primer lugar, porque de una cuestión individual pasamos a un asunto del ámbito de lo social; y, en segundo término, porque de lo anterior se derivan otras cuestiones epistemológicas y éticas. ¿Cómo pasar de lo individual a lo colectivo?; o sea: de una persona concreta a una construcción social basada en su identidad sexual. Más cuando esa persona ya ha fallecido y no puede tomar decisiones sobre si quiere o no ser nombrado y etiquetado. ¿Qué puede implicar todo ello, ya no para su vida sino para su representación social?, ¿qué opinaría la población sobre el nombrar, sin eufemismos, algo que ellos no nombraban?, ¿'mi respuesta narrativa influiría en el devenir de la investigación?, ¿y en el lugar?

\section{¿Por qué nombrar a los inmigrantes? (Barbara Rostecka)}

En este caso, la investigación que abrió la caja de los nombres se ha desarrollado entre 2007-2012 en la isla de Tenerife y se centraba en el asociacionismo de los inmigrantes no europeos. Eran tiempos bulliciosos para la inmigración masiva desde América Latina y África que apenas comenzaba a abrirse paso en este pequeño destino de las Islas Canarias. En aquella época apenas existían políticas de integración y de acogida y la población local, aunque de arraigada tradición emigratoria, miraba con recelo a sus nuevos vecinos. Era un tema nuevo, del que hablaban todos y no necesariamente bien. La prensa local estaba plagada de expresiones xenófobas y la investigación no salía del círculo de las políticas de la Unión Europea, marcadas por el tema del control de las fronteras y el imperativo de la integración. Casi todos investigaban la inmigración y lo hacían desde un paradigma miserabilista (Bourdieu, 1999). En palabras de mis entrevistados, debatir el tema de la inmigración era: "como darles pasto a las fieras. Porque se nos trata de una forma en un editorial y en otro también, que resulta difícil de entender de dónde puede salir tanto odio hacia el otro. Es odio hacia el otro" (Entrevista con Anahir Lema, líder de la Asociación Uruguaya en Tenerife. Realizada para proyecto de tesis de la autora). Ejemplos como éste, que denotaban con- frontación entre el discurso oficial sobre la inmigración y las reivindicaciones de los sujetos de mi investigación, se convirtieron para mí en "sucesos sensibilizadores", parafraseando a Blumer (1982). De repente y sin buscarlo me metí en una guerra que transcurría a nivel de una metacomunicación y donde tan a menudo me he sentido desafiada. Así en el verano del 2006 durante una concentración, convocada con el fin de recordar a los inmigrantes subsaharianos desaparecidos durante la travesía por el $\mathrm{mar}^{2}$, se me acercó uno de los líderes que organizaba el evento. Claramente disgustado por la escasa participación en la manifestación -que en el fondo pretendía desenmascarar la falsedad y crueldad de la gestión de las migraciones-, comenzó a decirme frases como: "¿Has venido a hacerte fotos con nosotros? ¿Dónde está tu jefe?, ¿¿dónde están todos los que cobran por trabajar con los inmigrantes?”. Lo decía desolado por su percepción del fracaso de una causa que, a pesar de ganarse votos como uno de los asuntos más importantes de la agenda política de aquella época, en la práctica se presentaba como una oportunidad para la mayoría de los agentes sociales: la de hacerse con un campo para realizar sus propios proyectos. Para aquel primer contrincante de mi método, yo era una representante más del Observatorio de la Inmigración de Tenerife (Obiten), que definía como un organismo alineado con las políticas oficiales de control y restricción de la inmigración e incapaz de hacer frente a la realidad. Según su opinión, la distancia que separa la investigación y la acción social, tal y como lo interpreté, convierte a los entes investigados en una especie de animal subyugado; y a mí, una pretendida investigadora objetiva, en una clase de garrapata social que se nutre y abastece de las cuestiones sociales, en vez de abrirles la puerta hacia la justicia.

Este inquietante sentimiento de ser responsable por la palabra, de entender que su uso levanta o tumba las dimensiones sociales y que el método define más bien las limitaciones del autor, lo he ido matizando a través del tiempo. Indudablemente reconozco la influencia del historiador colombiano Iván Forero Robayo, actualmente, Coordinador del Área de Estudios y Movimientos Sociales de la Comisión Española de Ayuda al Refugiado (CEAR) y Coordinador del III Foro Social Mundial de la Migración 
(FSMM). Tuve ocasión de hablar con él por primera vez durante el "Encuentro de Asociaciones y Redes de Personas Migrantes en el Archipiélago de Canarias”, en el otońo del 2008. Le estuve preguntando sobre los detalles de la organización del encuentro y, cuando le expuse mi interés por las asociaciones de los inmigrantes, me respondió que esperaba que pudiera hablar sobre las oportunidades que representan y no solo de las limitaciones del movimiento. Este mensaje lo repetía en todos sus discursos que pude escuchar: "el ser social necesita, también, de ternura para poder desarrollar todas sus virtudes". Se refería a la necesidad de desarrollar nuevas conexiones humanas que reconozcan la universalidad del sufrimiento humano y la necesidad de fe en uno mismo para que pueda superar situaciones difíciles, recuperar el valor, la dignidad y el sentido de la vida. Claramente, este último contrastaba con la cruel búsqueda de la objetividad que tan a menudo establecemos a través de una crítica arrolladora a los esfuerzos ajenos y que solemos dejar en modo anónimo. Yo entendí desde el primer momento que hablar de las limitaciones de las organizaciones inmigrantes, además de obvio, origina consecuencias difícilmente reconciliables con los valores de cualquier investigador social. A ninguno de nosotros nos proporcionaría satisfacción convertirse en esos labradores de lo social que fortalecen "estrategias adaptativas, confrontacionales, de marginación, indiferencia, etcétera, para llevar a cabo sus proyectos" (Thayer, 2009, 18).

Mi trabajo se centró en la visualización de esta pequeña fracción del asociacionismo inmigrante, del despertar de su reivindicación en medio del Océano y el campo social que ha creado. Nombrar a todos estos líderes ha sido apenas una consecuencia lógica de un constante diálogo con mis entrevistados que con tanta claridad demandaban ser reconocidos. Mi decisión no venía dada por la duda acerca de mi misión científica o si me comprometía o identificaba con la causa. Partía del claro discernimiento del momento en que pude desmarcarme de las formas modernas de ejercer el poder. Es decir, nombré y firmé con nombre y apellido las palabras de mis entrevistados en reconocimiento del esfuerzo y del valor de proyecto de la identidad inmigrante.

\section{Discusión}

La discusión se puede articular a través de dos elementos clave: la relación entre la elección de los procedimientos predeterminados y la forma de conocimiento lograda, por un lado, y el rol del investigador en los procesos de nombramiento/anonimización, por otro lado. Si aceptamos la premisa de que la forma epistemológica que adopta la investigación, lejos de ser una mera herramienta de representación de lo observado, es un sensible instrumento de construcción social (Ibáñez, 1994), la dialéctica entre lo nombrado y lo innombrable se convierte en una circunstancia susceptible de ser descrita y analizada. De hecho, la ontología y la epistemología ya se ocuparon de estas cuestiones al diferenciar las metodologías cuantitativas y cualitativas. Por tanto, en la medida en que estamos dispuestos a reflexionar sobre la forma en que el nombre propio condiciona el devenir de saberes populares (Fals, 2015) o sujetos emergentes y su relación con los esfuerzos emancipatorios de colectivos en situación de desventaja social (Fraser, 2008), podemos ubicarnos más claramente en estos asuntos éticos del quehacer del investigador/a social con sus luces y sombras.

\section{Anonimato: ¿protocolo para asegurar la objetividad $y$ proteger a los investigados?}

El inicio de nuestras investigaciones, como ocurre con una mayoría de trabajos de este tipo, partió de un presupuesto que solíamos tener interiorizado: anonimizar cualquier aportación. Es decir "expresar un dato relativo a entidades o personas eliminando la referencia a su identidad"3. Se asume que esto se debe, en parte, a la promulgación de la justa y necesaria ley sobre la protección de datos personales para proteger a los informantes de los riesgos que puede ocasionar su identificación. Ejemplo de ello son los numerosos programas, proyectos y guías centradas en las políticas de investigación en ciencias sociales: programas como el MOST $^{4}$ que aborda los dilemas de los investigadores sociales frente a asuntos éticos, legales, obligaciones y conflictos de intereses; las recomendaciones de las asociaciones internacionales de sociología 
y antropología $\mathrm{a}^{5}$, etc. El desarrollo de este tipo de regulaciones, que tienen el objeto de garantizar y proteger todo lo que concierne al tratamiento de los datos personales, las libertades públicas y los derechos fundamentales de las personas físicas, han articulado cuestiones del anonimato desde el campo jurídico al social, introduciendo nuevas y complejas dimensiones.

El primer ejemplo descrito hace entrever, como plantea Xochtil (2016), que la "justicia epistémica" es un arma de doble filo. Efectivamente, esta investigadora mexicana ha advertido del peligro de querer preservar todo tipo de datos en tiempos de conflictos, exponiendo a los sujetos investigados a determinados peligros. Ciertamente, los procedimientos de anonimización por razones de seguridad pueden parecer excepcionales, pero habría que pensar en los numerosos riesgos (individuales y colectivos) que pueden sufrir los participantes en las investigaciones. En la indagación sobre la alfarería, una información de índole íntima -como es la condición sexual del alfarero-en determinado tipo de sociedades puede repercutir no sólo en la memoria del maestro sino en el colectivo de alfareros que habían sido sus discípulos y que desarrollaban sus vidas y oficio en el lugar.

Sin embargo, tampoco hay que olvidar que la mayor parte de las investigaciones se han desarrollado bajo la constante vigilancia del enfoque positivista y de sus supuestos acerca de los papeles de investigador e investigado en los estudios científicos (Sztompka, 2002). Por eso, determinadas defensas a ultranza sobre el derecho a la intimidad, la garantía de sinceridad, protección y libertad de las personas entrevistadas u observadas lleva, en realidad, a la separación de los participantes del proceso de investigación y del control sobre la elaboración de los datos. Esos matices sugieren la necesidad de explorar la práctica del anonimato desde ángulos diferentes. En primer lugar, debe cuestionarse la efectividad del anonimato en su tarea de proteger aspectos relacionados tanto con la información como con el/los informantes. De hecho, ninguna precaución parece suficiente para evitar por completo cualquier divulgación deliberada o accidental de datos a partir de los cuales pudiese identificar a los participantes. Iphofen (2009) diferencia entre confidencialidad y anonimato. Mientras la primera sería una variable continua en la información y que tiene muchos grados de relevancia y/o evidencia/banalidad, el anonimato sería una variable dicotómica: identificar o no. Es decir, el proceso de anonimización no siempre preserva la confidencialidad, como lo muestra -de modo especial- la fotografía y el cine. Pero, en segundo lugar, algunos investigadores han cuestionado la idea de que los participantes siempre quieran ser anónimos (Tilley y Woodthorpe, 2011). Ser reconocidos, de diversos modos, puede ser un objetivo latente en informantes/entrevistados o incluso un acicate para participar en una investigación; también puede ser una forma de que los sujetos conserven-en cierto modo- la propiedad de sus historias (Asch, 1992; Grinyer, 2002) o como quiere Xochtil (2016), que se cultive la "justicia epistémica". En tercer lugar, Corden y Sainsbury (2006) advierten que nombres ficticios y sus modificaciones pueden tener efectos igualmente conflictivos, ya que distorsionan significados al añadir connotaciones falsas a personas y/o hechos, además de las dificultades que implica la elección de un nombre. Por último, tal y como se ha mencionado arriba, el desarrollo exponencial de una legislación y un aparato administrativo relacionado con el anonimato, imprescindible para que se cumpla la cada vez más compleja normativa sobre la protección de datos, introduce nuevos conflictos. En la actualidad, con el impresionante desarrollo de los Big Data se ańaden nuevos dilemas como el de la limitación al acceso y tratamiento de datos que sufren los investigadores. Algo similar ocurre con los abusos cometidos por parte de empresas especializadas en comerciar con datos personales y en manipular audiencias, mayormente a través de las redes sociales como ha sido el caso de Facebook y los escándalos políticos con los que ha sido vinculada dicha red.

\section{Nombrarlidentificar como criterio de validez}

Otro de los aspectos metodológicos que con frecuencia se relaciona con la cuestión de la identificación/anonimato es el concepto de validez, entendido en los estudios cualitativos como una adecuada representación de las construcciones mentales que los investigados ofrecen al investigador (Cortés, 1997). Y este se mostró con toda 
agudeza en la segunda de las investigaciones citadas. Describir nuevas formulaciones acerca de la inmigración, dentro del paradigma de la miserabilidad, era una tarea de por sí difícil ya que requería romper con marcos teóricos dominantes. Dejar sin firma estas reformulaciones significaba restarles credibilidad, dejar todo discurso en la nebulosa de la especulación de la investigadora y exponerles así -aún más- a la tan afamada capacidad de los sociólogos de reducir y destruir la emergencia de la vida social (Elías, 1991). Hay que recordar que, durante mucho tiempo, la noción de validez sirvió como estímulo a la exuberante proliferación de métodos cuantitativos y de arma contra el enfoque cualitativo (Altheide y Johnson, 1994). La visión postpositivista plantea que una investigación tendrá un alto nivel de validez en la medida en que sus resultados reflejen una imagen lo más completa, clara y representativa de la realidad o de la situación estudiada. Para ello, los estudios cualitativos han ahondado tanto en la metodología de recogida de información (largas estancias de convivencia con los observados, multiplicidad de puntos de vista, etc.), como en las técnicas de análisis (constante revisión, adecuación de las categorías a los participantes, continua actividad de realimentación y reevaluación, etc.). Este proceso ha tenido hitos relevantes. Así, en el periodo tradicional, marcado por la Escuela de Chicago, diversos estudios plantean crear recursos metodológicos capaces de detectar y de denunciar las situaciones de marginalidad originadas en la industrialización (Pineau y Marie-Michèle, 1983). En este contexto surgió la práctica de "nombrar" (identificar a las personas, hacerlas visibles o llamarlas por su nombre) a los sujetos que participaban en la investigación, objeto o/y sujeto de estudio, con el fin de documentar de la mejor manera los cambios sociales. Así surgió el método de los documentos personales. Sus creadores Thomas y Znaniecki, a raíz de su estudio sobre los emigrantes polacos en los Estados Unidos, publicado entre 1918 y 1920, defendían que los datos culturales están siempre ligados a los significados que les dan personas concretas, denominando a este rasgo como "el coeficiente humano" (Plumer, en el prólogo de Thomas y Znaniecki, 2006). Más adelante, en los años cincuenta, Fals Borda empeza- rá a concebir una serie de herramientas y métodos para redefinir el rol del campesino, defendiendo en sus investigaciones -realizadas en Colombia y otros países de América Latina-que se trata de un grupo plenamente capacitado para promover cambios sociales y organizativos (Moreno Moreno, 2017). Otra vertiente metodológica del acercamiento entre investigador e investigado ha partido de la reflexividad de diversas herramientas; por ejemplo, la realizada por Briggs (1986) sobre la entrevista, aunque concluya que es un potencial poco aprovechado por los investigadores debido a sus carencias en la preparación y desarrollo de esta técnica.

Esta noción de validez se relaciona con la de confiabilidad que ha representado siempre un requisito difícil para las investigaciones cualitativas, debido a la imposibilidad de repetir, stricto sensu, el mismo estudio. En todo caso, para sortear esta dificultad se ha recomendado aumentar el control del papel del investigador y la retroalimentación con los investigados (Le Compte y Goetz, 1982). Recordemos que la validez en los estudios interpretativos no se relaciona con la "capacidad para controlar el ambiente" sino con la facultad de propiciar el "crecimiento y desarrollo de seres humanos más autosuficientes en sentido pleno" (Martínez, 2004, p. 99) lo que une identidad y subjetividad.

\section{Construcción de la subjetividad y la identidad a partir de la identificación de los participantes}

Siguiendo a Derrida (2009), fue Nietszche, quién sembró la semilla de las políticas del nombre propio. Este término acentúa los procesos de subjetivación de los autores describiendo la escritura como un acto de autorreconocimiento, de reflexiones sobre el pasado, presente y futuro, y de posicionamiento social. Esto lleva a la delicada y autoexigente maestría de ser sincero a la hora de firmar palabras escritas por otros. Para Foucault (1984) la categoría del autor comenzó a delinearse cuando la historia de la cultura llegó a la moderna definición de la noción del sujeto, es decir, justo cuando la individualidad, la subjetividad y la originalidad comienzan a erigirse como valores culturales, sociales y políticos; por ello, la noción de autor conforma una unidad con su obra a través de su nombre. Es el 
nombre el que cataliza todos estos conceptos y posibilita el desempeño de las funciones sociales que, por un lado, elevan los textos al rango del discurso y, por otro, se incorporan en los circuitos de una determinada cultura. Ese nombre de autor más que una indicación, se erige como una previa clasificación y autorización para circular en el espacio disciplinado por las normas sociales. Esto lo acentúa Bourdieu (1984) para quien el autor no es una función, sino un sujeto que intenta hacerse con su propio campo dentro del entramado de las relaciones sociales y cuyo éxito se vincula con la formación del campo intelectual.

A diferencia del campo literario, los investigadores en las ciencias sociales no construimos nuestras obras a partir de biografías propias, sino que usamos las vivencias de otros para levantar piezas póstumas. Somos, pues, autores de las epistemes de los otros. Y ejercemos nuestras firmas con total libertad a pesar de que el construccionismo social desde Mead ha mostrado que las personas, o mejor las teorías con las que individualizamos a los sujetos individuales, son construcciones sociales e históricas (Gergen, 1985; Mead, 1934). ¿No deberíamos entonces revisar el grado de intrusismo de nuestras metodologías en tan moldeable materia social y nuestro papel en todo este proceso? La verdad es que lo han hecho varios autores. Elias (1991), autor incansable de la sociología histórica, definía a los sociólogos como mediadores de la palabra y del destino, afirmando que debemos tener claro que la función de los investigadores consiste en la nunca terminada tarea de descifrar los códigos de la opresión. Nombrar sin reducir o destruir sujetos frágiles es, según este autor, la tarea de acercar el sufrimiento humano con el fin de entender sus causas y así mitigar su prolongación. Fals Borda (2015) abogaba por la reorientación de las ciencias sociales hacia las necesidades de los grupos victimizados y subrayaba el papel de nombrar y validar que tienen los investigadores.

En Europa, fuera de los esfuerzos de algunos autores hacia las denominadas epistemologías del sur (Sousa, 2011), los debates siguen condicionados por las constricciones de las relaciones entre universidad y sociedad (Lanz, 2005). La categoría del autor sigue así reservada para un círculo estrecho de intelectuales. Sin embargo, es una cuestión que, con total obstinación y de múltiples formas, aparece durante el trabajo de campo. La subjetividad, al menos la de aquellos que no ostentan un estatus como escritor o publicista, puede salir de los silenciosos campos de lo íntimo.

\section{Identificación y simetría como democratización del espacio cientifico}

Debemos ser rigurosos en la exigencia de esos valores fundamentales sobre los que se asienta un Estado de Derecho: la igualdad, los derechos civiles y sociales, la no discriminación, la libertad de conciencia. A partir de estas premisas, pero sólo a partir de estas premisas, es posible el respeto a la diversidad cultural y religiosa que conlleva la inmigración. (...) Las facultades de ciencias juridicas y humanidades son las herramientas para la elaboración de propuestas y el desarrollo de los proyectos en este ámbito. (Luc André Diouf 6, X Jornada Técnica sobre la Inmigración organizada por Obiten, 02/12/2010)

La crisis de representación, en los ochenta del siglo XX, acentuó los dilemas de la investigación cualitativa y fundó las bases para una serie de revisiones de las políticas democráticas y de los debates sobre el futuro de la investigación social. A raíz de la creciente consciencia sobre los efectos del capitalismo en la sociedad, los presupuestos epistemológicos del método cualitativo han sido ampliados. El desarrollo de la reflexividad, a mediados del siglo XX, por los etnometodólogos americanos supuso un giro en la atención de los investigadores desde lo puramente descriptivo hacia la acción y la relación entre los hechos sociales. Desde entonces, sobre todo en contextos de marcada victimización de los grupos sociales, se abrieron vías académicas de validación de otras prácticas: este es el caso de Latinoamérica especialmente fructífera en este tipo de enfoques como el mencionado anteriormente de Fals Borda. En España, métodos como el participativo han sido empleados especialmente a partir de los años setenta (Colectivo IOÉ, 2003) y aplicados, 
sobre todo, por las Organizaciones no Gubernamentales bajo dos premisas: una, que la investigación en personas requiere de un compromiso ético capaz de promover el imperativo de igualdad (Giner, 1974; Plummer, 1983) y, dos, que la noción de democracia -tradicionalmente reservada para la actividad política- se asocie a este enfoque.

En los últimos decenios se han desarrollado nuevas metodologías como la investigación-acción-participación, planificación participativa, gestión compartida, entrevista y antropología reflexiva (Denzin, 2001; Strathern, 1987), antropología simétrica (Latour, 2007) o la antropología reversa (Wagner, 2010), entre otras. Un rasgo común a todas estas metodologías es reconocer que la información recolectada por los investigadores forma parte de un bien social, incluso moral de la comunidad y que sus usos van más allá de únicamente científico, estando conectados con el medio social en el que se desarrollan.

Sin embargo, también existen condiciones que llevan a los investigadores sociales a considerar la democratización de la investigación de forma mucho más universal. De hecho, el concepto de reflexividad es sumamente útil al respecto. Esta noción, cuyas raíces hemos señalado anteriormente, ha propulsado un giro en la forma de repensar la construcción de identidades ya que -hasta este momento- habían sido definidas a partir de rasgos particulares, como la etnicidad o el sexo, y más recientemente la nación. El "programa dialógico" propuesto por Alonso (1998) parte de la constatación de que los discursos, como modos de creación múltiple de lo social, encubren los mecanismos y formas emergentes de las relaciones humanas. En este sentido, habría que recordar varias acepciones de la reflexividad: la capacidad de una conversación interna con uno mismo, sobre la experiencia del proceso de investigación y acerca de su efectividad en la descripción de la realidad observada (Atkinson y Coffey, 2005; Briggs, 1986); una mirada hacia uno mismo, hacia el "self", hacia nuestro interior y hacia la exteriorización de nuestro "self, en lo que nos rodea (Carolan, 2003; De la Cuesta Benjumea, 2003). En ambos casos la reflexividad debe estar ligada a un dialogismo radical hacia la palabra nativa o al relato de los otros, dónde atendemos a sus nociones, sin suprimirlas, desmentirlas o ampliarlas.

\section{Volviendo a la praxis de lo social}

Volviendo a las investigaciones esbozadas al comienzo, los cuestionamientos y respuestas han variado a lo largo del tiempo. En el caso de la primera investigación, la confidencialidad parecía un asunto de relevancia. Desde el ámbito académico, cuando se nos solicita que explicitemos la metodología de nuestras investigaciones en realidad se refieren a cuantificar el esfuerzo y el seguimiento de las normas del denominado método científico. Sin embargo, se da escasa importancia a otros tipos de validaciones como los cuestionamientos de los paradigmas operantes y la reflexividad desarrollada a partir de estos, la comunicación continuada, la involuntaria o la metacomunicación (Briggs, 1986), etc., que surgen no tanto a partir de la aplicación voluntaria de técnicas como de la convivencia con los sujetos de estudio. La confidencialidad y sus usos es un claro ejemplo. De la misma surgen diversos tipos de información (Iphofen, 2009): alguna es mundana y no necesaria de mantenerse en privado; otra, al contrario, es altamente confidencial. De hecho, plantear como dilema el nombrar o no nombrar - identificar una práctica relacionada con la homosexualidad - ya desvela percepciones y valoraciones sociales que convierten una elección sexual en un tabú. ¿Era relevante la veracidad o no de un hecho o, al contrario, lo era la construcción y dinámicas sociales del mismo? Teoría, dilemas éticos y construcción social de la realidad pueden entrar en conflicto ¿Cómo percibe la gente del lugar, los vecinos, que los otros relacionen a su personaje más valorado con la homosexualidad (ellos ya lo hacían a través de los eufemismos)?, ¿podemos los de afuera hacer uso de la confidencialidad, de la confianza generada (no ya en el proceso de indagación sino en el de comunicación involuntaria), para nombrar lo que ellos - al menos abiertamente - no nombran?, ¿qué hubiera decidido el propio personaje? Sin embargo, los interrogantes nos llevaban más allá del propio alfarero ¿Qué pasaría con sus alumnos y, en especial, con los principales discípulos que continuaron con su taller y labor? ¿Importaba su opinión? Con el paso del tiempo y las reflexiones compartidas con los informantes se abrió paso otra sensación: la de la posible banalidad del debate si 
desde el comienzo hubiésemos utilizado un enfoque etnográfico más simétrico y reflexivo. Explicar el mismo proceso supuso identificar, sin nombrar, a su protagonista. Sin ser nombrado, quien primero lo reconoció fue su propia comunidad; el entorno exterior ya lo había valorado sobradamente por su labor artística: por tanto, nombrar la alfarería y al mejor alfarero era dar su identidad y la del lugar. Se decidió nombrar al alfarero porque parecía imposible no nombrar a alguien tan singular, al que todos nombraban. Sin embargo, también se optó por no identificar a otros protagonistas contemporáneos, como sus alumnos, aunque las razones y soluciones dadas para nombrar (sin identificar) a estos segundos, generaron también numerosas dudas. Primero porque el origen de los interrogantes sobre el nombramiento de los alumnos se centraba, de nuevo, en un círculo conformado por el alfarero-la homosexualidad-los discípulos y la interpretación del mismo por parte de la sociedad local; no nombrar significó para la investigadora una forma de preservar a determinados sujetos de posibles interpretaciones con tintes homófobos. Por tanto, se tomó decisiones ante la falta de atrevimiento versus discreción para preguntar algo que se consideraba dentro de la intimidad de las personas implicadas. Sin embargo, esta misma opción demuestra que es el investigador/a el que -en última instancia- dirime asuntos del nombramiento y el anonimato; por tanto, siempre tenemos que tener en cuenta las desigualdades y relaciones de poder donde generalmente el investigador/a conserva el papel de "director de orquesta” (Reygadas, 2014).

En cuanto a la segunda investigación, el aprendizaje se centró en los procesos de democratización de la investigación y la subjetivación, así como los controles de validez en este tipo de estudio desde el punto de vista técnico. De hecho, la demanda de la ternura en la mirada científica va más allá de la capacidad de introducir las emociones en una intervención social de investigación. Se precisa de una voluntad transformadora ante grupos sociales en desventaja social. Ante sus quejas, es absolutamente imprescindible que cambiemos nuestra mirada, que aprendamos a ver la realidad desde una perspectiva nueva para poder así sentir no solo el dolor de otras personas, sino el valor social de su ahogo.
Todos estos cuestionamientos no son inocentes ni banales porque perfilan la toma de decisiones del entrevistado, las opciones democratizadoras de la representación y la protección-física o social- del informante para que no sea identificado/a. En ocasiones, no dejamos ni que el dilema llegue a este punto: ya está decidido con anterioridad por nuestros intereses, sean personales o - mayormente - académicos. Porque el anonimato puede ser también otra forma de ocultar, de invisibilizar o de acallar autocontestaciones a los discursos que los mismos informantes expusieron (pasado) y que se plasma en escrituras con marcos bien determinados: por ejemplo, antes del texto literal del informante/ texto literal/ después del texto literal del informante; el antes y el después tienen una alta densidad interpretativa, de reelaboración que muchos investigadores evitan (evitamos) debatir y democratizar.

\section{Conclusiones}

El objetivo de este trabajo se nutre del contraste entre la tradición del anonimato y la reciente emergencia de nuevas tácticas: las que problematizan las practicas metodológicas surgidas en torno a la identidad y la representación de los sujetos en el proceso de investigación. Este enfoque, que dirige la mirada hacia nuestras propias experiencias investigadoras, se apoya tanto en la necesidad de una autobiografía crítica respecto a nuestra posición como investigador/a (Eyles y Smith, 1988), como en un enfoque integral (Guber, 2005) que incluya las implicaciones del trabajo de campo y los desplazamientos que se realiza desde lo teórico a lo empírico y viceversa. Desde este punto de vista, ejemplos y campos de análisis salen de las delimitaciones físico-temporales, ubicándose en experiencias del desafio surgidas a raíz de la representación (Maxwell, 1996; Nast, 1994; Katz, 1994). Los breves ejemplos expuestos visibilizan los dilemas éticos que conlleva el clásico método de distanciamiento, define la importancia de los contextos de nombrar y, por ende, de las estrategias de representación de la identidad/alteridad.

Como conclusión, se fijan argumentos en torno a los dos vértices clave en este análisis: el teórico-metodológico y el ético. Desde el primero, queda 
patente que la cuestión del anonimato, y la subsiguiente obligatoriedad de la protección de datos, no puede plantearse rígidamente, sino que, al contrario, la flexibilidad de procedimientos posibilita controles sobre la validez y confiabilidad de los estudios sociales. En cuanto al segundo argumento no habría que hablar de procedimientos nuevos, porque algunos han sido recuperados del olvido. Hemos recordado que ya en el año 1918, Thomas y Znaniecki nombraban a los protagonistas de sus historias, aunque en estos inicios el dilema de la identificación no requería tanto de un posicionamiento ético del investigador como de un claro posicionamiento epistemológico y ontológico. Así, el "factor humano", según Znaniecki consiste en dar prioridad a la experiencia del sujeto, captar en sus propios términos los significados que comparte con su grupo. Solo recientemente la idea de rescatar a los sujetos sociales se ha convertido en un imperativo de tipo moral para los investigadores comprometidos que postulan una "sociología de la liberación” (Fals, 2015). En todo caso, habría que reconocer que el debate sobre "nombrar o no nombrar" ocupa poco espacio en la producción social actual. Sobre el anonimato de nuestros informantes hay opiniones divergentes. Ciertamente, parece haber quedado atrás la etapa en la que usábamos camuflaje para nombrar las comunidades en las que trabajábamos y... ¿sería hoy posible con las búsquedas en red? Quizás siga siendo actual la recomendación de Fabian (1991) de analizar caso por caso: unos donde prevalecen los derechos de privacidad de nuestros informantes, otros dónde estamos obligados a reconocer su ayuda, uso de fuentes, etc., pero, también aquellos que no son cuestión de derechos u obligaciones, sino de ética sobre las consecuencias negativas de nombrar a nuestros informantes, sea por la presión social o la misma opresión política. En ocasiones el anonimato no beneficia a los enmascarados sino a los que enmascaran (Castillo, 2007).

En todo caso, queda patente, que la elección requiere de una nueva imaginación socioantropológica, capaz de prever nuevos efectos sociales de la investigación que van más allá de guardar el silencio para proteger a los investigados. Un foco relevante es el método etnográfico y la relación entre investigación-investigador-sujetos de estudio. Si partimos de que la etnografía supone la mediación de marcos de significado que entran en contacto durante el trabajo de campo y que, en ocasiones, estos saltan en pedazos, su posible sutura dependerá de un dialogo más radical que incluya la visibilidad de quiebras y conflictos de valores entre diferentes metodologías, autobiografías críticas y etnografías más simétricas.

\section{Notas}

1 Quiebra epistemológica en el sentido de Agar (1992), o sea una falta de concordancia que surge en el trabajo de campo entre el investigador, el contexto y las expectativas contenidas en los esquemas mediante los cuales organizamos nuestra propia experiencia.

2 Canarias es un puente hacia Europa para inmigrantes africanos que llegan aquí en pequeñas embarcaciones. Sólo en el 2007 en el mar rumbo a Canarias han muerto 1.260 inmigrantes, según la Guardia Civil, aunque Organizaciones No Gubernamentales triplican esta cifra.

3 Véase la Real Académica Española (RAE), en https:// www.fundeu.es/recomendacion/anonimizar/

4 Management of Social Transformations, programa de la Unesco creado en 1994 para promover políticas relacionadas con la investigación en las ciencias sociales. Disponible en http://www.unesco.org/new/es/social-and-human-sciences/themes/most-programme/ national-committees/ Consulta: 18/02/2018

5 En la actualidad son numerosas las recomendaciones y guías, en especial en el ámbito anglosajón y americano: The British Sociological Association Code of Ethical Practice, disponible en http://www.britsoc.co.uk/media/27107/StatementofEthicalPractice.pdf, el Comité de Ética de la American Anthropological Association, disponible en: http://www.aaanet.org/cmtes/ethics/ index.cfm, y otras. Consulta: 18/02/2018

6 Nació en 1965 en Joal-Fadiouth, Senegal. Hasta 2013 ocupó la Secretaría de migraciones de CCOO en Canarias, presidente de la comisión permanente del Foro canario de la inmigración (2000 a 2011), experto en la comisión de cooperación internacional al desarrollo (2000 a 2008) del Gobierno de Canarias, Miembro del Consejo superior de los senegaleses del exterior del Gobierno de Senegal (2000 a 2012).

\section{BIBLIOGRAFÍA}


AGAR, Michael. (1992), "Hacia un lenguaje etnográfico”, en C. Geertz, J. Clifford y C. Reynoso (Coord.), El surgimiento de la antropología posmoderna, Barcelona, Gedisa.

ALARCÓN, Luis. (2001), "Perspectivas de la sociología latinoamericana”. Cinta de Moebio, 11: 85-103.

ALONSO, Luis Enrique. (1998), La mirada cualitativa en sociología: una aproximación interpretativa. Madrid, Fundamentos.

ALTHEIDE, David L. y JOHNSON, John, M. (1994), "Criteria for Assessing Interpretative Validity in Qualitative Research", in Denzin, y Lincoln (eds.), Handbook of Qualiative Research, Londres, Sage.

ASCANIO-SÁNCHEZ, Carmen (2007), Género, tradición e identidades. Estrategias de Creación de valor en la alfarería de La Atalaya (Gran Canaria), Islas Canarias, Anroart.

ASCH, Timothy A. (1992), "The ethics of ethnographic film-making”, en Crawford y Turton (eds.), Film as ethnography, Manchester, University Press.

ATKINSON, Paul y COFFEY, Amanda. (2005), Encontrar el sentido a los datos cualitativos: Estrategias complementarias de investigación. Traducción de Eva Zimerman, 3a edición, Alicante, Universidad de Alicante.

BECK, Ulrich. (2004), Poder y contrapoder en la era global. Traducción de R. S. Carbó, Barcelona, Paidós.

BLUMER, Herbert. (1982), Interaccionismo Simbólico: Perspectiva y método. Barcelona, Ed. Hora.

BOURDIEU, Pierre. (1984), Campo del poder y campo intelectual. Buenos Aires, Folios.

BOURDIEU, Pierre. (Coord.) (1999), La miseria del mundo. Askal, Madrid

BOURDIEU, Pierre. (2011), "La ilusión biográfica”. Acta Sociológica, 5: 121-128.

BRIGGS, Charles. (1986), Learning how to ask. A sociolinguistics appraisal of the roll of the interview in social science research. Cambridge, Cambridge University Press.

CARENS, Joseph H. (2004), "A Contextual Approach to Political Theory". Ethnical Theory and Moral Practice, 7, 2: 117-132.

CAROLAN, Mary. (2003), "Reflexivity: A perso- nal journey during data collection". Nurse Researcher, 10, 3: 7-33.

CASTILLO, Alejandra. (2007), Julieta Kirkwood: Las politicas del nombre propio. Santiago de Chile, Palinodia.

COLECTIVO IOÉ. (2003), "Investigación acción participativa: propuesta para un ejercicio activo de la ciudadanía”, en Conferencia: Encuentro de la Consejería de

JUVENTUD. CÓRDOBA, junio 2003.

CORDEN, Anne. y SAINSBURY, Roy. (2011), "Using verbatim quotations in reporting qualitative social”. Social Policy Research Unit, University of York, York.

CORTÉS CAMARILLO, Graciela. (1997), "Confiabilidad y validez en estudios cualitativos". Educación y Ciencia, Nueva época, 1, 15: 77-82.

DE LA CUESTA BENJUMEA, Carmen. (2003), "El investigador como instrumento flexible de indagación”. International Journal of QualitativeMethods, 2 ,4: 50-77.

DERRIDA, Jacques. (2009), Otobiografias. La enseñanza de Nietzsche y la politica del nombre propio. Buenos Aires, Amorrortu.

DENZIN, Norman K. (2001), "The reflexive interview and a performative social science". Qualitative Research, 1, 1: 23-46.

DUBET, François. (2007), La experiencia sociológica. Traducción de Margarita Polo, Barcelona, Gedisa.

DUSSEL. ENRIQUE. (1988): "Introducción a la Filosofía de la Liberación”. Nueva América. Bogotá.

ELÍAS, Norbert. (1991), Mozart: sociología de un genio. Traducción de Marta Fernández-Villanueva y Oliver Strunk, Barcelona, Península.

ELÍAS, Norbert. (1994), Conocimiento y poder. Traducción de Julia Varela, Madrid, La Piqueta.

EYLES, John y SMITH, David M. (1988), Qualitative Methods in Human Geography. Oxford-Cambridge, Polity Press and Blackwell.

FABIAN, Johannes. (1991), Time and the Work of Anthropology. Critical Essays 1971-1991. Chur, Harwood Academic Publisher.

FALS BORDA, Orlando. (2015), "Retorno al compromiso práctico", en Herrera Farfán, y 
López Guzmán,(Comp.), Ciencia, compromiso y cambio social, Orlando Fals Borda, Antología, Argentina, Editorial El Colectivo.

FLICK, Uwe. (2004), Introducción a la investigación cualitativa. Madrid, Morata.

FOUCAULT, Michel. (1984), “¿Qué es un autor?".Dialéctica. Revista de la Escuela de Filosofia y Letras, 16: 23.47.

FRASER, Nancy. (2004), "La justicia social en la era de la política de identidad: Redistribución, reconocimiento y participación". Revista de Trabajo, 6: 56-81.

FREIRE, Paulo. (1988), ¿Extensión o Comunicación? La concientización en el medio rural. Siglo XXI. México.

GERGEN, Kenneth. J. (1985), “The Social Constructionist Movement in Modern Psychology". American Psychologist, 40, 3: 266-275.

GINER, Salvador. (1974), El progreso de la conciencia sociológica. Barcelona, Península.

GINER, Salvador. (2006), "Hannah Arendt: La primacía moral de la política”. Claves de Razón Práctica, 168: 69-98.

GRINYER, Anne. (2002), “The Anonymity of Research Participant: Assumptions, Ethic and Practicalities". Social Research Update, 36: 56.78, University of Surrey.

GUBER, Rosana. (2005), El salvaje metropolitano. Paidós, Buenos Aires.

IBÁÑEZ, Jesús. (1994), El regreso del sujeto: La investigación social de segundo orden. Madrid, Siglo XXI Editores.

IPHOFEN, Ron. (2009), "Ethical decision making in qualitative research". Qualitative Research, 11: 443-446.

KATZ, Cindi. (1994), "Playing the field: questions of fieldwork". Geography Professional Geographer, 46, 1: 67-72.

LANZ, Rigoberto. (2005), "En verdad, la universidad no se reforma”. Voltairenet.org, 28 de Febrero. Disponible enhttp://www.voltairenet.org/ article123931.html, consultado 30/12/2018.

LATOUR, Bruno. (2007), Nunca fuimos modernos: Ensayo de antropología simétrica. Madrid, Siglo XXI Editores.

LE COMPTE, Margaret D. y GOETZ, Judith P. (1982), "Problems of reliability and validity of ethnographic research". Review of Educational Research, 52, 1:31-60.

MARTÍNEZ MIGUELEZ, Miguel. (2004), Ciencia y Arte en la metodología cualitativa. México, Trillas.

MARRE, Diana. (2010), "Antropología y reproducción: las prácticas y/o la ética" en Del Olmo (coord.), Dilemas éticos en antropología: Las entretelas del trabajo de campo etnográfico, Madrid, Editorial Trotta.

MAXWELL, Joseph A. (1996), Qualitative research design: An interactive approach. Londres, Sage.

MEAD, George. (1934), Mind, Self, and Society: From the Standpoint of a Social Behaviorist. Chicago, University of Chicago Press.

MORENO MORENO, Mónica. (2017). "Construyendo una noción de campesinos con un método". Revista Latinoamericana de Metodología de las Ciencias Sociales, 7, 2: 8-28. Disponible en: http://www.memoria.fahce.unlp.edu. ar/art_revistas/pr.8171/pr.8171.pdf., consultado el 13/12/2018.

NAST, Heidi. (1994), "Women in the Field: Critical Feminist Methodologies and Theoretical Perspectives". The Professional Geographer, 46, 1: 54-66.

PINEAU, Gaston y MARIE-MICHÈLE. (1983), Produiresa vie: Autoformation et autobiogrphie. Montreal, Editorial Saint-Martin.

PLUMMER, Ken. (1983), Documents of Life. Londres, Allen.

POLANYI, Michael. (1966), El estudio del hombre. Buenos Aires, Paidós.

POPPER, Karl. (1977), Búsqueda sin término: una autobiografía intelectual. Traducción de Carmen García Trevijano, Madrid, Tecnos.

REYGADAS, Luis. (2014), "Todos somos etnógrafos. Igualdad y poder en la construcción de conocimiento antropológico", en Oehmichen Bazan (Ed), La etnografía y el trabajo de campo en ciencias sociales, México, Universidad Nacional Autónoma de México, 91-118.

REVILLA, Juan Carlos. (2003), "Los anclajes de la identidad personal”. Athenea Digital, 4: 54-67.

ROSTECKA, Barbara (2013), Campo sociocultural en torno a la inmigración extraeuropea en las Islas Canarias. Agentes, redes e identidades, Universidad de La Laguna, Tesis doctoral. https:// 
dialnet.unirioja.es/servlet/tesis?codigo $=158406$

SOUSA SANTOS, Boaventura de (2011), "Epistemologías del sur". Revista Internacional de Filosofía Iberoamericana y Teoría Social, 54: 17-39.

SZTOMPKA, Piotr. (2002), Sociología del cambio social. Traducción de Piotr Sztompka, Ángel Rivero Rodríguez, Madrid, Alianza.

STRATHERN, Marilyn. (1987), "Out of context: the persuasive fictions of anthropology [and comments and reply]". Current Anthropology, 28, 3: 251- 281.

THAYER CORREA, Luis E. (2009), La expropiación del tiempo y la apropiación del Espacio: $\mathrm{La}$ incorporación de los inmigrantes latinos a la Comunidad de Madrid. Tesis Doctoral. Universidad Complutense de Madrid.

THOMAS, William y ZNANIECKI, Florian. (2006), El campesino polaco en Europa y en América. Traducción de María Teresa Casado. Madrid, CIS.

TILLEY, Liz. y WOODTHORPE, Kate. (2011), "Is it the end for anonymity as we know it? A critical examination of this ethical principle of anonymity in the context of 21 st century demands on the qualitative researcher". Qualitative Research, 11, 2: 197-212.

TOURAINE, Alain. (2002), A la búsqueda de sí mismo: Diálogo sobre el sujeto. Barcelona, Paídos.

WAGNER, Roy. (2010), A invençao da cultura. Sao Paulo, CosacNaify.

XOCHTIL, Leyva Solano. (2016), "Pueblos en resistencia, justicia epistémica y guerra”. Cuadernos de Antropología Social, 44: 37 -50. 


\section{AS POLÍTICAS DO NOME PRÓPRIO: DILEMAS DE REPRESENTAÇÁO NAS CIÊNCIAS SOCIAIS}

\section{Carmen Ascanio-Sánchez e Barbara Rostecka}

Palavras-chave: Políticas do nome próprio; Dilemas éticos; Identidade/alteridade; Metodologias qualitativas; Subjetividade.

O objetivo deste artigo é contribuir para o debate sobre a representação dos sujeitos de estudo nas Ciências Sociais e, especificamente, os relacionados com as políticas do nome próprio e, portanto, do anonimato / identificação ou nomeação. Debatem-se, pois, as raízes teóricas de tal prática e suas implicaçóes, tanto na reformulação do método científico como nos sujeitos de estudo e políticas de representação. Tal é feito através de duas investigaçóes empíricas realizadas pelas autoras, onde essas questóes exigiram um foco especial. Na discussão explicitam-se as divergências existentes, a utilidade e/ ou os usos da nomeaçấo nos processos de democratizaçáo da investigação e, em particular, a necessidade de uma nova reflexão ligada a um diálogo mais radical entre quebras no conhecimento, autobiografias críticas e propostas etnográficas emergentes.

\section{THE POLITICS OF THE PROPER NAME: DILEMMAS OF REPRESENTATION IN THE SOCIAL SCIENCES.}

\section{Carmen Ascanio-Sánchez and Barbara Rostecka}

Keywords: Proper naming politics; Ethical dilemmas; Identity/alterity; Qualitative methodologies, Subjectivity.

This paper contributes to the debate surrounding the representation of study subjects in Social Sciences, looking specifically at how this relates to the politics of the proper name and, thus, of anonymity vs identification or naming. We discuss the theoretical roots of this practice, as well as its implications for the reformulation of the scientific method, for study subjects, and the politics of representation. The contribution is based on two empirical studies conducted by the authors in which these matters required specific attention. The discussion clarifies existing divergences, the utility and/ or use of naming in democratization processes in research and, in particular, the need to engage in a form of reflection linked to a more radical dialogue between breakdowns in understanding, critical autobiographies and emerging ethnographic approaches.

\section{LES POLITIQUES DU NOM PROPRE : DILEMMES DE REPRÉSENTATION DANS LES SCIENCES SOCIALES}

\author{
Carmen Ascanio-Sánchez et Barbara \\ Rostecka
}

Mots-clés: Politiques du nom propre; Dilemmes éthiques; Identité/altérité; Méthodologies qualitatives; Subjectivité.

L'objectif de cet article est de contribuer au débat sur la représentation des sujets d'études en sciences sociales et, plus précisément, ceux liés aux politiques du nom propre et, par conséquent, à l'anonymat/l'identification ou à la nomination. Par conséquent, les racines théoriques de cette pratique et ses implications sont débattues, tant dans la reformulation de la méthode scientifique que dans les sujets d'étude et les politiques de représentation. Cela se fait à travers deux enquêtes empiriques menées par les auteurs, où ces questions ont exigé une attention particulière. Dans la discussion, les divergences existantes, l'utilité et/ou l'utilisation de la nomination dans les processus de démocratisation de la recherche et, en particulier, la nécessité d'une nouvelle réflexion liée à un dialogue plus radical entre les ruptures de connaissances, les autobiographies critiques et les propositions ethnographiques émergentes. 


\section{LAS POLÍTICAS DEL NOMBRE \\ PRÓPRIO: DILEMAS DE \\ REPRESENTACIÓN EN LAS \\ CIENCIAS SOCIALES}

\section{Carmen Ascanio-Sánchez y Barbara Rostecka}

Palabras clave: Políticas del nombre propio, dilemas éticos, identidad/alteridad, metodologías cualitativas, subjetividad.

El objetivo de este artículo es contribuir al debate sobre la representación de los sujetos de estudio en las ciencias sociales y, concretamente, aquel relacionado con las políticas del nombre propio y, por tanto, del anonimato/ identificación o nombramiento. Se debaten las raíces teóricas de dicha práctica y sus implicaciones, tanto en la reformulación del método científico como en los sujetos de estudio y las políticas de representación. Todo ello a través de dos investigaciones empíricas, realizadas por las autoras, dónde estos asuntos requirieron de especial atención. En la discusión se explicitan las divergencias existentes, la utilidad y/o usos de nombrar en procesos de democratización de la investigación $y$, en especial, la necesidad de una nueva reflexividad ligada a un dialogo más radical entre quiebras del conocimiento, autobiografías críticas y propuestas etnográficas emergentes. 